\title{
Erratum to: Theoretical Calculations of the Surface Tension of Liquid Transition Metals
}

\author{
FATHI AQRA and AHMED AYYAD
}

DOI: $10.1007 / \mathrm{s} 11663-011-9487-4$

(C) The Minerals, Metals \& Materials Society and ASM International 2011

Erratum to: METALLURGICAL AND MATERIALS

TRANSACTIONS B, 2011, Vol. 42B, pp. 5-8.

DOI: $10.1007 / \mathrm{s} 11663-010-9456-3$

THE printer regrets an error in Table 1 of this paper.

The correct Table 1 is as follows:

Table I. Parameters Needed for Calculating Surface Tension of Metals. Calculated and Experimental Surface Tension Values of Some d-Block Metals

\begin{tabular}{|c|c|c|c|c|c|c|c|c|c|}
\hline Metal & $\begin{array}{l}\text { Atomic } \\
\text { Weight }\end{array}$ & $\begin{array}{l}\text { Melting } \\
\text { Point } \\
\left(\mathrm{K} /{ }^{\circ} \mathrm{C}\right)\end{array}$ & $\begin{array}{c}\text { Density } \\
\text { at Melting } \\
\text { Point }\left(\mathrm{g} / \mathrm{cm}^{3}\right)\end{array}$ & $\begin{array}{c}\text { Molar } \\
\text { Volume } \\
\left(\mathrm{cm}^{3} / \mathrm{mol}\right)\end{array}$ & $\begin{array}{l}\text { Sublimation } \\
\text { Energy } \\
(\mathrm{kJ} / \mathrm{mol})\end{array}$ & $f$ & $\begin{array}{c}\varphi \\
\left(\times 10^{-16}\right)\end{array}$ & $\begin{array}{c}\text { Calculated } \\
\text { Surface Tension } \\
\left(\mathrm{mJ} / \mathrm{m}^{2}\right)\end{array}$ & $\begin{array}{c}\text { Experimental } \\
\text { Surface Tension } \\
\left(\mathrm{mJ} / \mathrm{m}^{2}\right)\end{array}$ \\
\hline $\mathrm{Ti}$ & 47.86 & $1941 / 1668$ & 4.11 & 11.64 & 470 & 0.287 & 7.86 & 1380 & 1500 \\
\hline $\mathrm{Zr}$ & 91.224 & $2128 / 1855$ & 5.8 & 15.73 & 609 & 0.287 & 9.61 & 1461 & 1480 \\
\hline $\mathrm{Fe}$ & 55.845 & $1811 / 1538$ & 6.98 & 8.0 & 418 & 0.287 & 6.12 & 1560 & 1830 \\
\hline $\mathrm{Co}$ & 58.933 & $1768 / 1495$ & 7.75 & 7.60 & 428 & 0.287 & 5.92 & 1668 & 1830 \\
\hline $\mathrm{Ni}$ & 58.693 & $1728 / 1455$ & 7.81 & 7.52 & 430 & 0.287 & 5.87 & 1810 & 1838 \\
\hline $\mathrm{Pd}$ & 106.42 & $1828 / 1555$ & 10.38 & 10.25 & 377 & 0.287 & 7.22 & 1205 & 1475 \\
\hline $\mathrm{Pt}$ & 195.078 & $2041 / 1768$ & 19.77 & 9.87 & 566 & 0.287 & 7.042 & 1855 & 1860 \\
\hline $\mathrm{Cu}$ & 63.546 & $1357 / 1084$ & 8.02 & 7.92 & 338 & 0.287 & 6.1 & 1200 & 1310 \\
\hline $\mathrm{Ag}$ & 107.868 & $1235 / 962$ & 9.32 & 11.57 & 285 & 0.287 & 7.82 & 841 & 910 \\
\hline $\mathrm{Au}$ & 196.967 & $1337 / 1064$ & 17.31 & 11.38 & 368 & 0.287 & 7.74 & 1100 & 1149 \\
\hline $\mathrm{Zn}$ & 65.39 & $693 / 420$ & 6.57 & 9.95 & 130 & 0.240 & 7.08 & 854 & 789 \\
\hline $\mathrm{Cd}$ & 112.411 & $594 / 321$ & 7.996 & 14.05 & 112 & 0.240 & 8.91 & 584 & 637 \\
\hline $\mathrm{Hg}$ & 200.59 & $234 /-39$ & 13.534 & 14.82 & 61 & 0.210 & 9.24 & 428 & 438 \\
\hline
\end{tabular}

FATHI AQRA, Associate Professor, University Teacher, and Researcher, and AHMED AYYAD, Assistant Professor, University Teacher, and Researcher, are with the Department of Chemistry, Faculty of Science and Technology, Hebron University, Hebron, West Bank, Palestine. Contact e-mail: fathiaqra2009@hotmail.com

The online version of the original article can be found under doi:10.1007/s11663-010-9456-3.

Article published online February 8, 2011. 